\title{
Haemophilia, state of the art and new therapeutic opportunities.
}

\author{
Francesca Tomeo ${ }^{1}$, Segundo Mariz ${ }^{2}$, Angelo Loris Brunetta ${ }^{3}$, Violeta Stoyanova-Beninska ${ }^{4}$, \\ and Armando Magrelli ${ }^{5}$ \\ ${ }^{1}$ University of Rome Tor Vergata \\ ${ }^{2}$ European Medicines Agency \\ ${ }^{3}$ Italian Foundation 'L.Giambrone' for the cure of Thalassemia \\ ${ }^{4}$ Medicines Evaluation Board \\ ${ }^{5}$ Istituto Superiore di Sanita
}

July 25, 2020

\begin{abstract}
Haemophilia A and B are rare bleeding disorders. Over the past decades, they have been transformed from debilitating diseases to manageable conditions. However, several challenges and unmet needs remain in the treatment of the haemophilia limiting the QoL of the patients. These challenges are now being addressed by EHL recombinant factors, rebalancing and substitution therapies. Gene therapy and genome editing show promise for a definite clinical cure. Herein, we provide an overview of new therapeutic opportunities for haemophilia and their advances and limitations. The database on human medicines from EMA was used and data from rare disease (orphan) designations and EPARs were retrieved for the analysis. Clinical Trial databases were used to query all active studies on haemophilia. Gene therapy medicinal products based on AAV and lentiviral vectors are in development and clinical trials have reported substantial success in ameliorating bleeding tendency in haemophilia patients. The prospect of gene editing for correction of the underlying mutation is on the horizon with considerable potential. We are entering an era of innovation and abundance in treatment options for those affected by bleeding disorders but issues still remain about the affordability and accessibility to patients, the long-term durability, safety and efficacy.
\end{abstract}

\section{Haemophilia, state of the art and new therapeutic opportunities}

Francesca Tomeo ${ }^{1}$, Segundo Mariz ${ }^{2}$, Angelo Loris Brunetta ${ }^{3}$, Violeta Stoyanova-Beninska ${ }^{4}$ and Armando Magrelli ${ }^{5^{*}}$

${ }^{1}$ University of Rome Tor Vergata, Italy;francesca.tomeo@gmail.com

${ }^{2}$ Orphan Office, European Medicines Agency,Segundo.mariz@ema.europa.eu

${ }^{3}$ Italian Foundation 'L.Giambrone' for the cure of Thalassemia;lorisbrunetta@gmail.com

${ }^{4}$ Medicines Evaluation Board, The Netherlands;v.stoyanova@cbg-meb.nl

5 National Center for Drug Research and Evaluation, Istituto Superiore di Sanità, Italy ;armando.magrelli@iss.it

* Correspondence: armando.magrelli@iss.it; Tel.: +390649904148

Keywords: Haemophilia; replacement therapy; rebalancing therapy; gene therapy; orphan drugs; orphan designation; marketing authorization; EMA; advanced therapies.

Word count, table count, figure count $-\mathbf{4 7 9 2}$ words, 5 tables, 3 figures. 


\begin{abstract}
Haemophilia A and B are rare bleeding disorders. Over the past decades, they have been transformed from debilitating diseases to manageable conditions. However, several challenges and unmet needs remain in the treatment of the haemophilia limiting the QoL of the patients. These challenges are now being addressed by EHL recombinant factors, rebalancing and substitution therapies. Gene therapy and genome editing show promise for a definite clinical cure. Herein, we provide an overview of new therapeutic opportunities for haemophilia and their advances and limitations. The database on human medicines from EMA was used and data from rare disease (orphan) designations and EPARs were retrieved for the analysis. Clinical Trial databases were used to query all active studies on haemophilia. Gene therapy medicinal products based on AAV and lentiviral vectors are in development and clinical trials have reported substantial success in ameliorating bleeding tendency in haemophilia patients. The prospect of gene editing for correction of the underlying mutation is on the horizon with considerable potential. We are entering an era of innovation and abundance in treatment options for those affected by bleeding disorders but issues still remain about the affordability and accessibility to patients, the long-term durability, safety and efficacy.
\end{abstract}

\title{
1. Introduction
}

Haemophilia A and B are rare X chromosome-linked monogenic disorders resulting from a deficiency in a coagulation factor in the intrinsic pathway of blood coagulation. These two diseases affect approximately 210,000 persons worldwide [1]. Developing medicines intended for small numbers of patients has little commercial incentive under normal market conditions. Therefore, the EU offers a range of incentives to encourage the development of designated orphan medicines [2]. The scope of this review is to provide an overview of the clinical development of medicinal products currently holding an orphan designation in EU for treatment of haemophilia A and B based on current experience and knowledge.

Haemophilia is caused by mutations in the genes encoding for Factor VIII (haemophilia A, the more prevalent form) and Factor XI (haemophilia B), resulting in decreased production and/or function of factor VIII and factor IX proteins. Both FVIII and FXI are naturally synthesized in the liver: FVIII in the liver sinusoidal endothelial cells (LSEC) and FIX in hepatocytes. Patients with severe haemophilia have an absence of circulating plasma FVIII and FIX activity $(<1 \%)$, resulting in spontaneous or excessive bleeding into joints and muscles. If left untreated, recurrent bleedings result in the development of chronic arthropathy (knee, ankle and elbow) and early mortality from spontaneous or trauma-induced bleeds.

Haemophilia care has undergone remarkable improvements over the past decades and haemophilia has been transformed from a debilitating disease to a manageable condition. Although the diffused/pervasive availability of safe and effective replacement therapy, haemophilia patients continue to experience enormous burden of treatment, spontaneous bleeding and progressive joint disease, as well as high rates of development of inhibitors to factor products. In the past 20 years, recombinant bioengineering has led to extended half-life (EHL) therapies with increased efficacy and easier modes of administration, as well as decreased immunogenicity. [4]. We are now on a paradigm shift in prophylaxis for haemophilia through the development of the first substitution therapy, as well as innovative rebalancing therapies. Investigational gene therapy holds the promise for a definitive clinical cure [5-6].

\subsection{Prevalence of Haemophilia in EU}

In order to obtain prevalence information for individuals living with haemophilia A and B in Europe, relevant information from the World Federation of Haemophilia (WFH) annual global surveys and the literature were reviewed. To estimate the prevalence of haemophilia throughout Europe, in general, the WFH 2018 survey was utilized [1]. For countries where prevalence was not available from the 2018 survey, the most recent version of the WFH report that contained the relevant data was used.

Table 1 presents data of the haemophilia A and B prevalence for the European countries who reported data to the WFH. According to the consolidated WFH survey data, the prevalence among European countries ranged from 0.27 per 10,000 males in Finland to 1.38 per 10,000 males in Ireland for Haemophilia A and 0.06 per 10.000 males in Finland to 0.51 per 10.000 in Ireland. 
In a recent meta-analysis [7] it was shown that more than 1,125,000 men around the world have an inherited bleeding disorder; 418,000 of those have a severe version of haemophilia. Previously, only 400,000 people globally were estimated to have haemophilia. The results proposed an estimate of 2.46 cases per 10.000 males for all severities of haemophilia A and 0.5 cases per 10.000 males for all severities of haemophilia B. Applying a prevalence of 2.96 cases per 10.000 males for haemophilia A and B combined to the current European population estimate of 517 million (248 million males) (retrieved fromhttps://ec.europa.eu/eurostat/data/database , accessed in June 2020) generates an estimate of 73.408 males with haemophilia in EU, of whom about 16,120 should have severe haemophilia. These estimates are higher than those historically cited but still characterize haemophilia as rare diseases on the basis of definitions used in European Union $(<5$ cases per 10000 persons Regulation (EC) No. 141/2000 of the European Parliament and of the Council) [2].

\subsection{Treatments for Haemophilia}

The current 'gold-standard' treatment for haemophilia is prophylactic factor replacement therapy, intravenous injection of factor VIII/XI concentrates. There are many preparations available in Europe (Table $2)$.

Prophylaxis is used with the aim of rising FVIII and FIX activity above a level that is detectable ( $>1 \%)$ to prevent bleeding and reduce or delay the incidence of joint disease. The short biological half-lives of FVIII and FIX proteins require frequent infusions, three times a week (haemophilia A) or twice a week (haemophilia B) [8]. A major complication of factor replacement therapy is the formation of inhibitory antibodies against the coagulation factors, rendering these ineffective [9].

Inhibitors develop in approximately $25 \%$ to $30 \%$ of haemophilia A patients and, less frequently, in $3 \%$ to $5 \%$ of haemophilia B patients. Treatment with bypassing agents (BPAs) and immune tolerance induction (ITI) is required in such patients to eradicate inhibitors, nonetheless patients continue to exhibit increased morbidity and mortality [9-10]. In non-inhibitor patients, prophylaxis can decrease the frequency of bleeding and slow the progression of joint disease, thus improving the quality of life. However, studies have shown that bleeding events are not eliminated for all patients, and joint disease still appears in young adults [11-12].

The mainstay for treatment of bleeding episodes in patients with high-responding inhibitors is the so-called 'by-passing agents', recombinant FVIIa and anti-inhibitor coagulant complex (FEIBA). In patients with inhibitors to FIX, prophylaxis with FIX products is not possible.

Several challenges and unmet needs remain in the treatment of haemophilia, since patients continue to experience breakthrough bleedings, progressive joint disease, and high rate of inhibitor development. These numerous challenges can be addressed by emerging technologies such as gene replacement therapies (advanced therapy medicinal products or ATMPs).

\section{Materials and Methods}

The database on human medicines from European Medicines Agency was used and data from Rare disease (orphan) designations (https://www.ema.europa.eu/en/medicines/download-medicinedata\#rare-disease-(orphan)-designations-section) and European public assessment reports (EPARs) (https://www.ema.europa.eu/en/medicines/download-medicine-data\#european-public-assessment-reports(epar)-section) were retrieved (20 May 2020) for the analysis.

A control with the data obtained from EMA database was performed for consistency with the Community Register of orphan medicinal products from EC (https://ec.europa.eu/health/documents/communityregister $/ \mathrm{html} /$ reg_od_act.htm?sort=a) in which all ODs are registered. (20 May 2020)

The ClinicalTrials.gov database and The European Union Clinical Trials Register were used to query all active studies on Haemophilia (20 May 2020). ClinicalTrials.gov provides information on medical studies in human volunteers. Most of the records on ClinicalTrials.gov describe clinical trials. The EU Clinical Trials Register contains information on interventional clinical trials on medicines conducted in the European Union (EU), or the European Economic Area (EEA). 


\section{Results}

\subsection{Orphan designation of Haemophilia $A$ and $B$}

A new legislation was introduced by the EU in 2000 in order to provide incentives for the development of medicines for rare diseases called orphan medicinal products. Orphan designation (OD) through the European Orphan Regulation fosters research in new therapeutic approaches in the treatment of rare diseases and sets the criteria for receiving an OD. We retrieved the list of all rare disease (orphan) designations from 2000 (start of the Orphan regulation) to end of 2019 which are found on the EMA's website (www.ema.europa.eu). Resulting in a total of positive 2289 orphan designations. Data extraction forms were designed as: medicine name, active substance, Agency product number, date of first decision, disease/condition, EU designation number, status of orphan designation, first published, revision date, URL.

Among these orphan designations, we selected only ODs for the treatment of haemophilia A and B. 41 ODs resulted from the filtered research.

Among them, 14 medicinal products were withdrawn from the Community Register of designated Orphan Medicinal Products and 1 OD expired (Figure 1). Thus, 26 'active' ODs resulted from the research.

\subsubsection{Classification of Haemophilia ODs}

Of all active ODs, treatment of Haemophilia B and Haemophilia A are almost equally represented (54\% and $46 \%$ respectively). It was noted that factor replacement therapy medicinal products represent the largest group (42\%) followed by Gene Therapy Medicinal Products (GTMPs) and rebalancing therapy medicinal products (39\% and 19\% respectively) (Figure 2). Replacement therapy involves the supply of the missing clotting factor to the patient from an external source, which is slowly injected into a vein. Rebalancing therapy aims to restore or rebalance the coagulation system targeting natural anticoagulant pathways.

\subsection{Authorised medicinal products for Haemophilia}

In order to understand which products had received a central market authorisation for Haemophilia A and B we carried out an investigation in EMA's EPARs (European public assessment reports) database, which contains all scientific assessment reports of medicines authorised at a European Union level. We downloaded the table of all EPARs for human and veterinary medicines from EMA's website. A total of 1694 medicinal products resulted from the research. We selected only medicinal products for the treatment of haemophilia A and B in human. 24 medicinal products resulted from the research. Among them, two medicinal products (Iblias and Nonafact) were withdrawn from use in the European Union (both at the request of the marketing-authorisation holder). Currently, there are 22 authorised medicinal products for the treatment of haemophilia A and B. Table 3 provides a detailed list of the authorised medicines. Among them, only Alprolix and Idelvion were designated orphan medicinal products and received a 10-years market exclusivity. The remaining authorised medicines are no longer orphan medicines and were withdrawn from the Community register of orphan medicinal products at the time of the granting of a marketing authorisation. These products did not provide any advantage compared to the current state of art, and did not brought the innovation that was hoped to transform the treatment of patients with Haemophilia A and B.

\subsection{Novel therapeutic opportunities in haemophilia care}

Novel therapeutic opportunities to improve treatment of haemophilia patients are rapidly evolving. These include: novel FVIII/FIX products, substitution therapies, haemostatic rebalancing therapy and gene therapy/editing. Figure 3 provides an overview of the coagulation cascade and the site of action of novel therapeutics in haemophilia care.

\subsubsection{Recombinant and modified release FVIII and FIX products}

The short half-life of the standard therapies for haemophilia requires frequent administration within prophylaxis regimens. Extended half-life FVIII/FIX recombinant concentrates have been obtained by fusion to polyethylene glycol (FVIII \& FIX), IgG1-Fc (FVIII \& FIX) or albumin. These modifications have provided 
prominent decrease in injection frequency - every 1 to 2 weeks for prophylaxis [4]. EHL clotting factors increase treatment adherence, improve clinical outcomes and provide an opportunity for improved individualised treatment for haemophilia. Elocta and Alprolix, for haemophilia A and B respectively, are examples of EHL which have been recently authorised for the use in the European Union.

\subsubsection{Substitution therapy}

A subcutaneously administered bispecific monoclonal antibody (Emicizumab) has recently been developed. It bridges FX and FIXa and acts as a partial functional mimic to FVIIIa, to restore the missing function of FVIIIa. Because of its unique structure, Emicizumab is not expected to induce or be affected by factor VIII inhibitors. HAVEN 1, a phase 3 non interventional study in severe haemophilia A patients with inhibitors, showed an $87 \%$ reduction in bleed rates compared with no prophylaxis, and a $79 \%$ reduction compared with prior BPA prophylaxis [13]. HAVEN 3 study evaluated Emicizumab prophylaxis in haemophilia A patients without inhibitors, using weekly dosing, and demonstrated a $68 \%$ reduction in treated bleeds compared with prior factor VIII prophylaxis [14]. These results support the hypothesis that the level of steadystate maintenance of haemostasis achieved with Emicizumab prophylaxis can result in superior efficacy with respect to traditional prophylaxis.

\subsubsection{Rebalancing therapy}

Haemostasis is a complex physiological process that maintains a balance between the normal blood flow within the vasculature and the induction of blood clot formation following injury [15]. In the presence of a coagulation factor deficiency, such as Haemophilia, this delicate balance is shifted toward bleeding. In contrast, derangements in the natural anticoagulant pathways can lead to thrombosis. Evidences from current knowledge demonstrate that targeting these natural anticoagulant pathways (anti-thrombin and tissue factor pathway inhibitors, protein $\mathrm{S}$ and protein $\mathrm{C}$ ) can restore the haemostatic equilibrium in the presence of a bleeding disorder [16]. Fitusiran is an RNA interference (RNAi) therapy which targets antithrombin (AT) in the liver and interferes with AT translation by binding and degrading messenger RNA-AT to prevent AT synthesis and promote haemostasis. In both preclinical and clinical studies, AT knockdown led to dosedependent lowering of AT levels and reduced the bleeding phenotypes in haemophilia patients. [17-18].

\subsection{Emerging Advanced Therapeutic Medicinal Products: Gene therapy for haemophilia}

Gene replacement therapies represent a frequently submitted category of therapeutic for orphan designation [19]. In this form of ATMPs, a functional copy of a defective gene in the target condition, which is either absent or expressed as a non-functional protein is substituted by a fully functional gene which is inserted using a viral vector thereby offering a highly effective means for overcoming diseases, such as haemophilia. The gene correction in haemophilia is well suited because it is associated with a well understood mutated gene sequence which can be substituted by a corrected DNA sequence leading to the endogenous production of a functional factor VIII or IX [5]. This would therefore alleviate the need for exogenous sources of the defective clotting factors.

An important characteristic of haemophilia treatment is that there is no need to normalize circulating clotting factor levels to obtain a therapeutic effect since a slight increase in plasma clotting factor levels (above 1\%) is sufficient to decrease the risk of morbidity and mortality. As a consequence, a small increase in clotting factor levels by gene therapy can greatly improve the clinical symptoms. In addition, therapeutic efficacy of GTMPs can be determined by clear-cut clinical endpoints such as circulating clotting factor levels and bleeding frequency [20]. Thus, gene therapy could provide a continuous source of clotting factor from a single treatment.

Initial clinical gene therapy studies using integrating retroviral, adenoviral, and non-viral (ex vivo ) approaches were associated with transient low-level factor expression [21-22].

This led to a shift toward usage of 'non-integrating' recombinant adeno-associated viral (AAV) vectors. AAV vectors are derived from wild-type AAV [23], a member of the parvovirus family. Wild-type AAV is nonpathogenic, weakly immunogenic, and replication-deficient, requiring a helper virus for replication. AAV 
vectors can deliver a therapeutic transgene cassette up to $5 \mathrm{~kb}$ into both dividing and nondividing cells. The DNA sequences carried by recombinant AAV vectors are stabilised predominantly in an episomal form so that long-term expression can occur only with delivery into long-lived, post mitotic cell types; the vector DNA integrates at a very low frequency and is typically lost from replicating cells. The recombinant vector has tropism for a range of target tissues including the liver, cell types in the retina and the central nervous system, skeletal muscle, and cardiac muscle, among others. AAV vectors are the most frequently used viral vectors for gene therapy for Haemophilia (90\%), followed by lentiviral vectors (10\%).

Table 4 provides a detailed list of GTMPs for Haemophilia, which were granted an orphan designation by European Commission.

FVIII and FIX synthesis primarily takes place in the liver, from where the proteins can easily enter the bloodstream. This makes hepatocytes a suitable target for gene therapy in haemophilia.

Over the years, hepatic in vivo gene transfer using adeno-associated viral (AAV) vectors has shown the best success in preclinical and clinical studies, with several clinical studies for both hemophilia A and B enrolling patients for phase 3 trials. We analyzed the studies present in the Clinical trial.gov and in the EU Clinical Trials Register repositories [24-25].

Many AAV vectors have progressed into clinical studies for both haemophilia A and B. The ClinicalTrials.gov database presently lists 29 active gene therapy clinical trials for haemophilia, 12 clinical trials to evaluate different AAV-based GTMPs for haemophilia B and 11 trials to evaluate AAV-FVIII gene therapy for haemophilia A (Table 5), with some overlap, as the same vector is being evaluated in both phase $1 / 2$ and phase 3 studies.

Of the ongoing Haemophilia A and B AAV clinical trials, there are remarkable successes. The trial sponsored by St. Jude Children's Research Hospital haemophilia B patients, using an scAAV2/8-LP1-hFIXco vector, was the first long-term success story for haemophilia gene therapy [26].

However, an important limitation to successful AAV-base gene therapy is the pre-existing humoral capsid immunity. Immune recognition by cytotoxic $\mathrm{CD} 8^{+} \mathrm{T}$ cells or antibody responses to the vector capsid, the transgene product, or both can compromise the therapeutic expression of the transgene. Wild-type AAV infection occurs during childhood, and thus patients may develop neutralising antibiotics (nAb) that prevent gene transfer with AAV vectors. Cross-reactivity with multiple AAV serotypes has also been reported. As a result, many patients have been excluded from recent studies. The main toxicity observed in AAV-based clinical studies has been dose-related elevation of liver transaminase following vector infusion. Within some studies, this coincides with the demonstration of cell-mediated AAV capsid immunity. Most studies have used either early intervention or prophylaxis with corticosteroids in order to protect transduced hepatocytes. Although the majority of episodes have been managed effectively with this approach, some episodes have been associated with partial or complete loss of transgene expression, despite intervention [26].

Due to the pre-existing AAV humoral immunity and the potential loss of vector transduction with time, there is for the need of other viral and non-viral-based vectors for transgene delivery. Lentiviral vectors may complement the therapeutic reach of AAV vectors due to lower incidence of pre-existing humoral immunity and greater packaging capacity. Pre-clinical studies using in-vivo or ex-vivo stem cell (haematopoietic or induced pluripotent) transduction or blood outgrowth endothelial cells have been recently reviewed [27-28]. Two phase 1 studies are registered with plans to evaluate ex-vivo lentiviral stem cell transduction for FVIII (NCT03818763) or FVIII/FIX (NCT03217032/NCT03961243).

\subsection{Genome editing}

The most advanced approach to genome editing in haemophilia is usage of zinc finger nuclease (ZFN). There have been several recent orphan designations of products with this alternative approach to overcoming the defective gene. The gene editing technique can correct the endogenous genetic defect or direct the integration site of a therapeutic gene through nuclease-targeted double-strand DNA breaks and homologydirected repair. Thus, gene-editing technologies are based on harnessing the natural repair machinery of the 
cell to modify DNA. A phase 1 study (NCT02695160) is currently recruiting using AAV-directed correction using ZFN targeted insertion of FIX gene into the first intron of the albumin locus. Although gene editing holds significant promise for haemophilia, in its current state, the approach still requires AAV for delivery of the nuclease or transgene [29]. Therefore, the same limitations for AAV gene therapy are present for gene-editing approaches.

\section{Discussion}

Current haemophilia treatment consists of parenterally administered plasma-derived or recombinant clotting factor concentrates. Patients have significantly benefited from replacement therapy, as it greatly improves their quality of life and prolongs life-expectancy. It has been observed that the recently introduced into the market modified release formulations which can be applied sub-cutaneously have improved the dosing regimen for these patients.

Although current coagulation factor replacement prophylaxis regimens are effective, they are expensive, challenging to adhere to, result in regular sub-therapeutic trough factor levels, and are potentially immunogenic. As such, nearly all haemophilia patients would benefit from an intervention that induces stable factor expression. There is the need to develop such a therapy as the haemophilia population ages and develops medical co-morbidities that make effective prophylaxis administration more difficult.

In recent studies, gene therapies have induced sustained levels of FVIII and FIX, prevented bleeding, and reduced or eliminated the use of factor replacement products. This approach could also alleviate the heavy burden of the need for frequent factor infusions, concerns about inhibitor development, and limited global access to factor products. However, while short-term risks appear to be low, it has been noted that long-term safety and efficacy are not yet established.

Two separate AAV-based gene therapy phase 1/2 clinical trials for haemophilia A have now reported stable FVIII levels after a single vector dose, demonstrating that gene therapy is an effective investigational treatment of patients with adult haemophilia A. In haemophilia B, even though multiple phase $1 / 2$ gene therapy clinical trials, have now reported stable therapeutic FIX expression after a single treatment using an AAV vector.

Some additional considerations are related to the type of vector used (namely AAV-based or lentiviral) and their applicability in different age groups of patients with haemophilia. Humoral immunity to wild-type AAV represents one of the most important limitations to successful systemic transduction with AAV vectors. Preexisting and recall responses to the WT virus from which the vector is engineered, or to the transgene product, can interfere with therapeutic efficacy [30-31]. Humans are naturally infected with wild-type AAV during childhood, and thus may develop neutralising antibodies that prevent gene transfer with AVV vectors. Due to the high level of conservation in the amino acid sequence among AAV capsids, anti-AAV antibodies show cross- reactivity with multiple serotypes. This has resulted in exclusion of many patients from recent studies. The main toxicity seen in clinical trials has been dose-related elevation of liver transaminase following vector infusion and, in some studies, this coincides with the demonstration of cell-mediated AAV capsid immunity. Early intervention or prophylaxis with corticosteroids with the aim of protecting transduced hepatocytes have been used in most studies, but not all episodes have been managed effectively and partial/complete loss of transgene expression occurred. Pre-existing AAV humoral immunity and the potential loss of vector transduction with time represent important and limiting issues in the AAV-based gene therapy and can render the therapy ineffective. Thus, there is a requirement for other viral and non-viral-based vectors for transgene delivery. The predominant methods studied in Haemophilia have used lentiviral vectors, for which there is a lower incidence of pre-existing humoral immunity and which have a greater packaging capacity than AAV vectors.

A specific concern is the application of gene therapies in children. It is still unclear how effective AAV gene therapy will be in young children lacking data on long term exposure, particularly in terms of the risk for inhibitor and potential loss of factor expression over time as a result of dilution of AAV-transduced hepatocytes during liver growth [5]. Integrating Lentiviral Vector may provide an alternative vector platform 
for treating children with haemophilia with in vivo liver gene delivery of immune stealth and tolerogenic LV vectors to hepatocytes. In children and adults with either high-risk mutations for inhibitors, history of inhibitor, or presently with inhibitor, it is unclear whether the robust AAV mediated liver tolerance and ITI observed in haemophilia animal models will translate in humans [5].

Lentiviral vectors (LV) potentially offer certain advantages. They are able to efficiently integrate into the target genome, even when the cells are not actively dividing. If the liver is the target organ, this can lead to transduction of nondividing hepatocytes with the potential of long-term transgene expression. The liver also contains an abundance of antigen-presenting cells (APCs) that can also be transduced. Transduction of APCs might increase the risk of an immune response to FVIII or FIX and thereby inhibit long-term expression. The use of hepatocyte-specific promoters and incorporation of hematopoietic-specific microRNA target sequences into the vector may reduce this immune risk.

Another point of discussion is the external validity of the results obtained from the clinical trials with a selected patient population. Although the clinical data supporting gene therapy for haemophilia have been rather positive, the enrolled subjects bias are a selected group, and thus some caution is advised in extending the benefits of gene therapy to the general population of patients with haemophilia due to potential selection bias of the results from the clinical studies.

Finally, the many factors playing a role in the decision-making process for patients with haemophilia need to be considered carefully. Despite some very promising data provided on the novel treatments for haemophilia $\mathrm{A}$ and $\mathrm{B}$, patients still have needs that require clarifications in consideration of the impact that these new therapies can have on their lives. The use of Emicizumab in Haemophilia A patients with inhibitors constitutes a remarkable achievement as this novel medicine, approved in EU on January 2018, allow the patients to have one subcutaneous administration per week, rather than one every $2 / 3$ days. This is a great improvement in the treatment of Haemophilia A, especially in terms of QoL, but there's still the need to know how and when it will be possible to switch the patients with inhibitors to this novel approach. Gene therapies using the AAVs look very promising in reducing the need for replacement therapy very close to 0 as the ABR as well. But in this context, patients still have some concerns that need to be elucidated, particularly many unanswered questions about the AAVs, the increase of the liver enzymes level and hepatotoxicity, the reduction in the FVIII expression during the time and the possibility to be re-treated with the vector if a drop in the FVIII expression occurs. Further, some ethical considerations have to be done concerning the use of gene therapies in children. The hot topic for the patients' community is the current lack of long-term data on the treatment use of gene replacement and splicing therapies. The latter is an urgent challenge to be addressed in the upcoming months in order to allow haemophilia patients to be much more aware about the therapeutic options available for the whole community. Considering 1 million persons with haemophilia worldwide, of whom about 418000 have severe and mostly undiagnosed disease, this constitutes a challenging task for researchers and health care systems, especially because only 210,000 patients have been identified and reported globally (WHF report 2018). More efficient diagnostic approaches are needed in less developed countries to take advantage of current and future treatment modalities, including gene therapy.

\section{Conclusions}

Treatments have evolved regarding the management of the haemophilias. From a therapeutic approach focused just on filtered exogenous factor replacement, thinking has evolved to improve the delivery and pharmacokinetic properties of these exogenous replacement factors. Currently gene replacement/splicing advanced therapeutic medicinal products have emerged and represent a potential cure for people with haemophilia A and B.

In recent studies, gene therapies have induced sustained levels of FVIII and FIX, prevented bleeding, and reduced or eliminated the use of factor replacement products. This approach could also alleviate the heavy burden of the need for frequent factor infusions, concerns about inhibitor development, and limited global access to factor products. However, while short-term risks appear to be low, it has been noted that long-term safety and efficacy are not yet established. 
Although the clinical data supporting gene therapy for haemophilia have been rather positive, some caution is advised in extending the benefits of gene therapy to the general population of patients with haemophilia due to potential selection bias of the results from the clinical studies.

Careful consideration should be given to the advantages and disadvantages of the different type of vectors and their suitability for the different haemophilia patients depending on their age, availability or risk for inhibitors, and other factors.

All the above-mentioned factors play a role in the decision making for each individual patient with haemophilia. In addition to gene therapy, novel replacement therapies and other non-factor-based therapy options are being developed, with one such therapy being released in 2018. We are entering an era of innovation and abundance in treatment options for those affected by bleeding disorders. There are many questions that remain: what are the eligibility criteria to qualify for this therapy, will it be affordable and accessible to patients, what is the long-term durability and efficacy and is it safe? Although, the choice of therapy available in the future may in part be directed by financial implications, this may, with time, allow for more personalized care with improvement in quality of life for the next generation of patients with haemophilia.

\section{Acknowledgment*}

Conflict of interest statement: The authors declare no conflict of interest.

Funding: This research received no external funding.

Author Contributions: Conceptualization, V.S.B. and A.M.; formal analysis, F.T.,S.M.; writing —original draft preparation, FT.; writing - review and editing, F.T., S.M.,A.L.B.,V.S.B., A.M.; supervision, AM.; All authors have read and agreed to the published version of the manuscript.

\section{References}

1. World Federation of Haemophilia (2019) Report on the Annual Global Survey 2018. WFH, Montreal, Canada.http://www1.wfh.org/publications/files/pdf-1731.pdf. Accessed May 20, 2020

2. Regulation (EC) No. 141/2000 of the European Parliament and of the Council.https://eurlex.europa.eu/legal-content/EN/TXT/PDF/?uri=CELEX:32000R0141\&from=EN. Accessed May 20, 2020.

3. Giannelli F, Green PM. The molecular basis of haemophilia A and B. Baillieres Clin Haematol . 1996;9(2):211-228. doi:10.1016/s0950-3536(96)80059-x.

4. Weyand AC, Pipe SW. New therapies for hemophilia. Blood . 2019;133(5):389-398. doi:10.1182/blood2018-08-872291.

5. Perrin GQ, Herzog RW, Markusic DM. Update on clinical gene therapy for hemophilia. Blood . 2019;133(5):407-414. doi:10.1182/blood-2018-07-820720.

6. Pasi KJ, Rangarajan S, Mitchell N, et al. Multiyear Follow-up of AAV5-hFVIII-SQ Gene Therapy for Hemophilia A. N Engl J Med . 2020;382(1):29-40. doi:10.1056/NEJMoa1908490.

7. Iorio A, Stonebraker JS, Chambost H, et al. Establishing the Prevalence and Prevalence at Birth of Hemophilia in Males: A Meta-analytic Approach Using National Registries. Ann Intern Med . 2019;171(8):540-546. doi:10.7326/M19-1208.

8. Srivastava A, Brewer AK, Mauser-Bunschoten EP, et al. Guidelines for the management of hemophilia. Haemophilia . 2013;19(1):e1-e47. doi:10.1111/j.1365-2516.2012.02909.x.

9. White GC 2nd, Kempton CL, Grimsley A, Nielsen B, Roberts HR. Cellular immune responses in hemophilia: why do inhibitors develop in some, but not all hemophiliacs?. J Thromb Haemost . 2005;3(8):1676-1681. doi:10.1111/j.1538-7836.2005.01375.x,

10. Oldenburg J. Optimal treatment strategies for hemophilia: achievements and limitations of current prophylactic regimens. Blood . 2015;125(13):2038-2044. doi:10.1182/blood-2015-01-528414.

11. Mazepa MA, Monahan PE, Baker JR, Riske BK, Soucie JM; US Hemophilia Treatment Center Network. Men with severe hemophilia in the United States: birth cohort analysis of a large national 
database. Blood . 2016;127(24):3073-3081. doi:10.1182/blood-2015-10-675140.

12. Di Minno MND, Pasta G, Airaldi S, et al. Ultrasound for Early Detection of Joint Disease in Patients with Hemophilic Arthropathy. J Clin Med . 2017;6(8):77. doi:10.3390/jcm6080077.

13. Shima M, Hanabusa H, Taki M, et al. Factor VIII-Mimetic Function of Humanized Bispecific Antibody in Hemophilia A. N Engl J Med . 2016;374(21):2044-2053. doi:10.1056/NEJMoa1511769.

14. Roche's Hemlibra reduced treated bleeds by 96 percent compared to no prophylaxis in phase III HAVEN 3 study in haemophilia A without factor VIII inhibitors.https://www.roche.com/media/releases/medcor-2018-05-21.htm. Accessed on May 20, 2020.

15. Miyazaki, K. (2013) Coagulation of Blood. In: Gellman M.D., Turner J.R. (eds) Encyclopedia of Behavioral Medicine. Springer, New York, NY.

16. Shetty S, Vora S, Kulkarni B, et al. Contribution of natural anticoagulant and fibrinolytic factors in modulating the clinical severity of haemophilia patients. Br J Haematol . 2007;138(4):541-544. doi:10.1111/j.1365-2141.2007.06693.x.

17. Pasi KJ, Rangarajan S, Georgiev P, et al. Targeting of Antithrombin in Hemophilia A or B with RNAi Therapy. N Engl J Med . 2017;377(9):819-828. doi:10.1056/NEJMoa1616569.

18. Machin N, Ragni MV. An investigational RNAi therapeutic targeting antithrombin for the treatment of hemophilia A and B. J Blood Med . 2018;9:135-140. doi:10.2147/JBM.S159297.

19. Farkas AM, Mariz S, Stoyanova-Beninska V, et al. Advanced Therapy Medicinal Products for Rare Diseases: State of Play of Incentives Supporting Development in Europe. Front Med (Lausanne) . 2017;4:53. Published 2017 May 16. doi:10.3389/fmed.2017.00053.

20. Chuah MK, Evens H, VandenDriessche T. Gene therapy for hemophilia. J Thromb Haemost . 2013;11 Suppl 1:99-110. doi:10.1111/jth.12215.

21. Swystun LL, Lillicrap D. Gene Therapy for Coagulation Disorders. Circ Res . 2016;118(9):1443-1452. doi:10.1161/CIRCRESAHA.115.307015.

22. Chapin JC, Monahan PE. Gene Therapy for Hemophilia: Progress to Date. BioDrugs . 2018;32(1):9-25. doi:10.1007/s40259-017-0255-0.

23. Atchison RW, Casto BC, Hammon WM. Adenovirus-associated defective virus particles. Science . 1965;149(3685):754-756. doi:10.1126/science.149.3685.754.

24. ClinicalTrials.gov.https://clinicaltrials.gov/ct2/home. Accessed on May 20, 2020.

25. EU Clinical Trials Register.https://www.clinicaltrialsregister.eu/. Accessed on May20, 2020.

26. Nathwani AC, Reiss UM, Tuddenham EG, et al. Long-term safety and efficacy of factor IX gene therapy in hemophilia B. N Engl J Med . 2014;371(21):1994-2004. doi:10.1056/NEJMoa1407309.

27. Doshi BS, Arruda VR. Gene therapy for hemophilia: what does the future hold?. Ther Adv Hematol . 2018;9(9):273-293. Published 2018 Aug 27. doi:10.1177/2040620718791933.

28. Ide LM, Gangadharan B, Chiang KY, Doering CB, Spencer HT. Hematopoietic stem-cell gene therapy of hemophilia A incorporating a porcine factor VIII transgene and nonmyeloablative conditioning regimens. Blood . 2007;110(8):2855-2863. doi:10.1182/blood-2007-04-082602.

29. Sangamo Therapeutics (2018). Sangamo announces treatment of first patient in phase $1 / 2$ clinical trial of in vivo genome editing therapy for hemophilia B.https://investor.sangamo.com/news-releases/newsrelease-details/sangamo-announces-treatment-first-patient-phase-12-clinical-0. Accessed on May 20, 2020.

30. Herzog RW. Hemophilia Gene Therapy: Caught Between a Cure and an Immune Response. Mol Ther . 2015;23(9):1411-1412. doi:10.1038/mt.2015.135.

31. Mingozzi F, High KA. Immune responses to AAV vectors: overcoming barriers to successful gene therapy. Blood . 2013;122(1):23-36. doi:10.1182/blood-2013-01-306647.

Tables

Table 1. Haemophilia A and B prevalence in European Countries. 


\begin{tabular}{|c|c|c|c|c|c|}
\hline Country & Population & $\begin{array}{l}\text { People with } \\
\text { Hemophilia A }\end{array}$ & $\begin{array}{l}\text { Prevalence } \\
\text { (per 10000) }\end{array}$ & $\begin{array}{l}\text { People with } \\
\text { Hemophilia B }\end{array}$ & $\begin{array}{l}\text { Prevalence } \\
\text { (per 10000) }\end{array}$ \\
\hline Country & Population & $\begin{array}{l}\text { People with } \\
\text { Hemophilia A }\end{array}$ & $\begin{array}{l}\text { Prevalence } \\
\text { (per 10000) }\end{array}$ & $\begin{array}{l}\text { People with } \\
\text { Hemophilia B }\end{array}$ & $\begin{array}{l}\text { Prevalence } \\
\text { (per 10000) }\end{array}$ \\
\hline Albania & $2.876 .101,00$ & 161 & 0,56 & 33 & 0,11 \\
\hline Austria & $8.747 .358,00$ & 658 & 0,75 & 117 & 0,13 \\
\hline Belgium & $11.348 .159,00$ & 970 & 0,85 & 242 & 0,21 \\
\hline Bulgaria & $6.981 .642,00$ & 560 & 0,80 & 68 & 0,10 \\
\hline Cyprus & $1.172 .458,00$ & 43 & 0,37 & - & - \\
\hline Czech & $10.561 .633,00$ & 937 & 0,89 & 136 & 0,13 \\
\hline \multicolumn{6}{|l|}{ Republic } \\
\hline Denmark & $5.731 .118,00$ & 410 & 0,72 & 102 & 0,18 \\
\hline Estonia & $1.316 .481,00$ & 97 & 0,74 & 10 & 0,08 \\
\hline Finland & $5.495 .096,00$ & 150 & 0,27 & 33 & 0,06 \\
\hline $\begin{array}{l}\text { France } \\
\text { (metropolitan) }\end{array}$ & $66.896 .109,00$ & 5864 & 0,88 & 1498 & 0,22 \\
\hline Germany & $82.667 .685,00$ & 3686 & 0,45 & 628 & 0,08 \\
\hline Greece & $10.746 .740,00$ & 873 & 0,81 & 184 & 0,17 \\
\hline Hungary & $9.817 .958,00$ & 893 & 0,91 & 230 & 0,23 \\
\hline Ireland & $4.773 .095,00$ & 617 & 1,3 & 243 & 0,51 \\
\hline Italy & $61.680 .122,00$ & 3992 & 0,65 & 886 & 0,14 \\
\hline Latvia & $1.960 .424,00$ & 129 & 0,66 & 21 & 0,11 \\
\hline Lithuania & $2.872 .298,00$ & 147 & 0,51 & 24 & 0,08 \\
\hline Netherlands & $16.877 .351,00$ & 1026 & 0,61 & 125 & 0,07 \\
\hline Norway & $5.232 .929,00$ & 325 & 0,62 & 90 & 0,17 \\
\hline Poland & $37.948 .016,00$ & 2413 & 0,64 & 428 & 0,11 \\
\hline Portugal & $10.324 .611,00$ & 539 & 0,52 & 112 & 0,11 \\
\hline Romania & $19.705 .301,00$ & 1615 & 0,82 & 210 & 0,11 \\
\hline Slovakia & $5.428 .704,00$ & 521 & 1,00 & 79 & 0,15 \\
\hline Slovenia & $2.064 .845,00$ & 207 & 1,00 & 30 & 0,15 \\
\hline Spain & $47.042 .984,00$ & 1679 & 0,36 & - & - \\
\hline Sweden & $9.798 .871,00$ & 860 & 0,88 & 195 & 0,20 \\
\hline United & $65.637 .239,00$ & 6559 & 1,00 & 1518 & 0,23 \\
\hline Kingdom & & & & & \\
\hline
\end{tabular}

Table 2. Licensed products for the treatment of Haemophilia A and B.

\begin{tabular}{lll}
\hline Product type & Licensed products $^{\mathbf{1}}$ & Indication (abbreviated) $^{\text {Recombinant FVIII concentrate }}$ \\
(rFVIII) & Advate Adynovi Afstyla Elocta & Treatment and prophylaxis of \\
& Esperoct Jivi Kogenate Bayer & bleeding in patients with \\
& Kovaltry Helixate NexGen & haemophilia A (congenital factor \\
& NovoEight Nuwiq Recombinate & VIII deficiency) \\
Plasma-derived FVIII & ReFacto AF Vihuma Voncento & \\
concentrate (pdFVIII) & Beriate Haemoctin Hemofil & \\
& Immunate Monoclate Octanate &
\end{tabular}




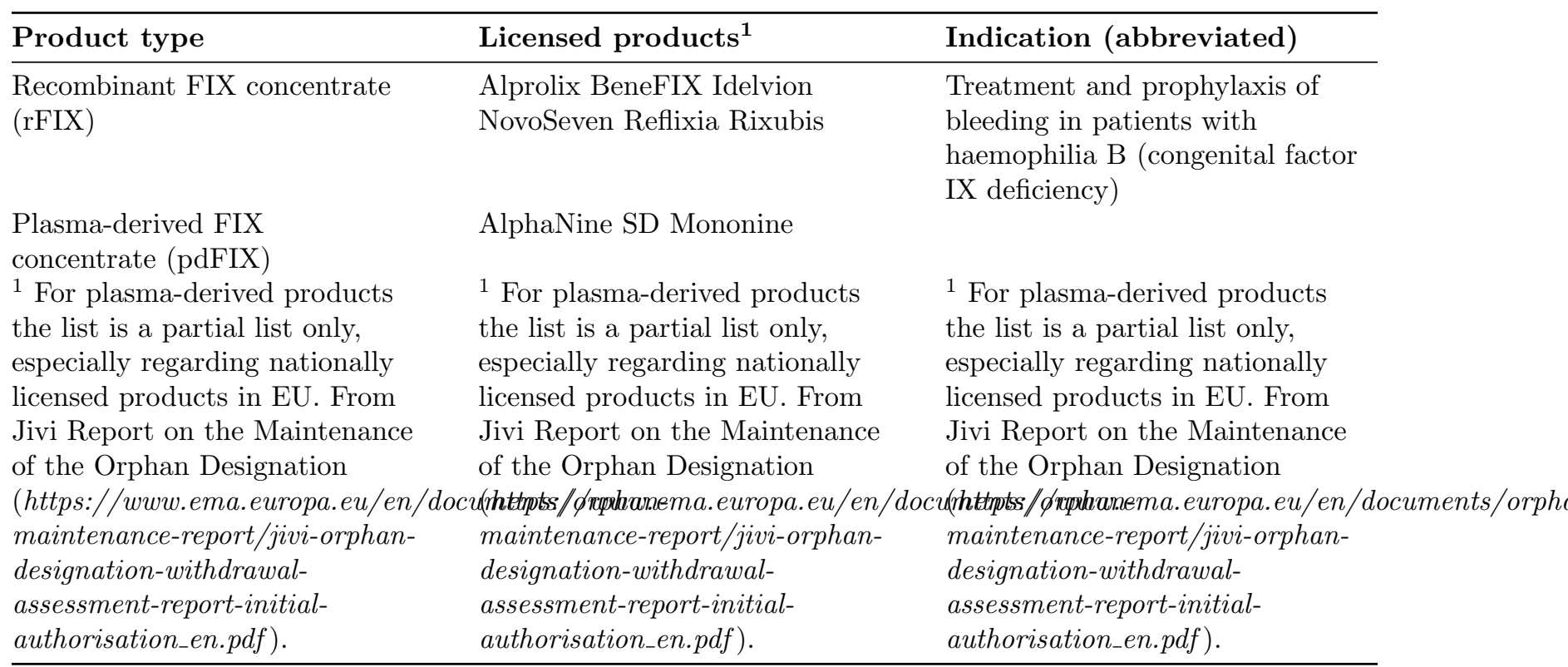

Table 3. List of centrally authorised medicinal products for the treatment of HA and HB.

\begin{tabular}{|c|c|c|c|c|}
\hline Medicine name & $\begin{array}{l}\text { International } \\
\text { non-proprietary } \\
\text { name (INN) }\end{array}$ & Therapeutic area & MA date & $\begin{array}{l}\text { MA holder/ } \\
\text { company name }\end{array}$ \\
\hline Esperoct & $\begin{array}{l}\text { Turoctocog alfa } \\
\text { pegol }\end{array}$ & Hemophilia A & $20 / 06 / 2019$ & $\begin{array}{l}\text { Novo Nordisk } \\
\mathrm{A} / \mathrm{S}\end{array}$ \\
\hline Jivi & $\begin{array}{l}\text { Damoctocog alfa } \\
\text { pegol }\end{array}$ & Hemophilia A & $22 / 11 / 2018$ & Bayer AG \\
\hline Hemlibra & Emicizumab & Hemophilia A & $23 / 02 / 2018$ & $\begin{array}{l}\text { Roche } \\
\text { Registration } \\
\text { Limited }\end{array}$ \\
\hline Adynovi & $\begin{array}{l}\text { Rurioctocog alfa } \\
\text { pegol }\end{array}$ & Hemophilia A & $8 / 01 / 2018$ & $\begin{array}{l}\text { Baxalta } \\
\text { Innovations } \\
\text { GmbH }\end{array}$ \\
\hline Refixia & $\begin{array}{l}\text { Nonacog beta } \\
\text { pegol }\end{array}$ & Hemophilia B & $2 / 06 / 2017$ & $\begin{array}{l}\text { Novo Nordisk } \\
\mathrm{A} / \mathrm{S}\end{array}$ \\
\hline Vihuma & Simoctocog alfa & Hemophilia A & $13 / 02 / 2017$ & Octapharma AB \\
\hline Afstyla & Lonoctocog alfa & Hemophilia A & $4 / 01 / 2017$ & $\begin{array}{l}\text { CSL Behring } \\
\text { GmbH }\end{array}$ \\
\hline Alprolix & Eftrenonacog alfa & Hemophilia B & $12 / 05 / 2016$ & $\begin{array}{l}\text { Swedish Orphan } \\
\text { Biovitrum AB } \\
\text { (publ) }\end{array}$ \\
\hline Idelvion & $\begin{array}{l}\text { Albutrepenonacog } \\
\text { alfa }\end{array}$ & Hemophilia B & $11 / 05 / 2016$ & $\begin{array}{l}\text { CSL Behring } \\
\text { GmbH }\end{array}$ \\
\hline Kovaltry & Octocog alfa & Hemophilia A & $18 / 02 / 2016$ & $\begin{array}{l}\text { Bayer Pharma } \\
\text { AG }\end{array}$ \\
\hline Elocta & Efmoroctocog alfa & Hemophilia A & $18 / 11 / 2015$ & $\begin{array}{l}\text { Swedish Orphan } \\
\text { Biovitrum AB } \\
\text { (publ) }\end{array}$ \\
\hline
\end{tabular}




\begin{tabular}{|c|c|c|c|c|}
\hline Medicine name & $\begin{array}{l}\text { International } \\
\text { non-proprietary } \\
\text { name (INN) }\end{array}$ & Therapeutic area & MA date & $\begin{array}{l}\text { MA holder/ } \\
\text { company name }\end{array}$ \\
\hline Obizur & Susoctocog alfa & Hemophilia A & $11 / 11 / 2015$ & $\begin{array}{l}\text { Baxalta } \\
\text { Innovations } \\
\text { GmbH }\end{array}$ \\
\hline Rixubis & Nonacog gamma & Hemophilia B & $19 / 12 / 2014$ & $\begin{array}{l}\text { Baxalta } \\
\text { Innovations } \\
\text { GmbH }\end{array}$ \\
\hline Nuwiq & Simoctocog alfa & Hemophilia A & $22 / 07 / 2014$ & Octapharma AB \\
\hline NovoEight & Turoctocog alfa & Hemophilia A & $13 / 11 / 2013$ & $\begin{array}{l}\text { Novo Nordisk } \\
\text { A/S }\end{array}$ \\
\hline Voncento & $\begin{array}{l}\text { Human } \\
\text { coagulation factor } \\
\text { VIII/human von } \\
\text { willebrand factor }\end{array}$ & $\begin{array}{l}\text { Hemophilia A, } \\
\text { von Willebrand } \\
\text { Diseases }\end{array}$ & $12 / 08 / 2013$ & $\begin{array}{l}\text { CSL Behring } \\
\text { GmbH }\end{array}$ \\
\hline Advate & Octocog alfa & Hemophilia A & $2 / 03 / 2004$ & $\begin{array}{l}\text { Takeda } \\
\text { Manufacturing } \\
\text { Austria AG }\end{array}$ \\
\hline Kogenate Bayer & Octocog alfa & Hemophilia A & $4 / 08 / 2000$ & Bayer AG \\
\hline Helixate NexGen & Octocog alfa & Hemophilia A & $4 / 08 / 2000$ & Bayer AG \\
\hline ReFacto AF & Moroctocog alfa & Hemophilia A & $13 / 04 / 1999$ & $\begin{array}{l}\text { Pfizer Europe MA } \\
\text { EEIG }\end{array}$ \\
\hline BeneFIX & Nonacog alfa & Hemophilia B & $27 / 08 / 1997$ & $\begin{array}{l}\text { Pfizer Europe MA } \\
\text { EEIG }\end{array}$ \\
\hline NovoSeven & $\begin{array}{l}\text { Eptacog alfa } \\
\text { (activated) }\end{array}$ & $\begin{array}{l}\text { Hemophilia B, } \\
\text { Thrombasthenia, } \\
\text { Factor VII } \\
\text { Deficiency, } \\
\text { Hemophilia A }\end{array}$ & $23 / 02 / 1996$ & $\begin{array}{l}\text { Novo Nordisk } \\
\text { A/S }\end{array}$ \\
\hline
\end{tabular}

Table 4. List of gene therapy medicinal products for haemophilia.

Active substance

Adeno-associated viral vector serotype 8 containing a functional copy of the codon-optimised F8 cDNA encoding the B-don Adeno-associated viral vector serotype 5 containing a B-domain deleted variant of human coagulation factor VIII gene

Recombinant adeno-associated viral vector containing a bioengineered capsid and a codon-optimised expression cassette to Recombinant adeno-associated viral vector serotype 6 encoding the B-domain-deleted human factor VIII

Adeno-associated viral vector serotype rh10 containing the human factor IX gene for the treatment of haemophilia B-

Fidanacogene elaparvovec

Lentiviral vector encoding human coagulation factor IX

Adeno-associated viral vector containing the human factor-IX gene

Recombinant adeno-associated viral vector containing a codon-optimized Padua derivative of human coagulation factor IX Recombinant adeno-associated viral vector serotype S3 containing codon-optimised expression cassette encoding human coa

Table 5. Summary of active gene therapy clinical trials for hemophilia registered on ClinicalTrials.gov. 


\begin{tabular}{|c|c|}
\hline NCT Number & Title \\
\hline CT04418414 & matopoietic Stem Cell Transplantation Gene Therapy for Treatment of Severe Hemophilia A \\
\hline CT03818763 & Gene Therapy Trial for Platelet Derived Factor VIII Production in Hemophilia A \\
\hline CT04370054 & Study to Evaluate the Efficacy and Safety of PF-07055480 in Moderately Severe to Severe Hemophilia $A$ \\
\hline ТT03001830 & Gene Therapy for Haemophilia A. \\
\hline CT03520712 & Gene Therapy Study in Severe Hemophilia A Patients With Antibodies Against AAV5 \\
\hline СТ03061201 & Study of Recombinant AAV2/6 Human Factor 8 Gene Therapy SB-525 in Subjects With Severe Hemop \\
\hline CT03003533 & A Gene Transfer Study for Hemophilia A \\
\hline NCT03370913 & Study To Evaluate The Efficacy and Safety of Valo \\
\hline CT03: & Safety and Dose Escalation Study of ar \\
\hline TCT02: & tudy in Severe Haem \\
\hline NCT04 & Study to Evaluate the Efficacy and Safety of Volactocoge \\
\hline TCTO: & to Evaluate The $\mathrm{E}$ \\
\hline TCTO & Delivers a \\
\hline $\mathrm{TCT} 03$ & Gene Therapy \\
\hline CT03 & Study of SPK-8016 Gene Therap \\
\hline CTO & A Study to \\
\hline CT0 & Theral \\
\hline CT04 & Gen \\
\hline 43 & Lentiviral FIX \\
\hline 03 & A Long-Term Follow-Up Study of Haemophilia B \\
\hline 342 & Trial of AAV5-hFIX in Seve \\
\hline 444 & A Factor IX Gene Therapy Str \\
\hline 980 & Long-term Safety and Efficacy Study of SPK-9001 i \\
\hline & A Phase 1/2 Study of SHP648, an Adeno-Associated V \\
\hline & Ascending Dose Study of Genome Editing by Zinc Finger Nuclease Therapeutic SB-FIX in Subjects Wit \\
\hline & \\
\hline NCT00979238 & Dose-Escalation Study Of A Self Complementary Adeno-Associated Viral Vector For Gene Transfer in H \\
\hline
\end{tabular}

\begin{tabular}{ll}
\hline NCT02971969 & Long-Term safety, Tolerability, and Efficacy of DTX 101 (AAVrh10FIX) in Adults with Moderate/Severe t \\
\hline NCT03489291 & Dose Confirmation Trial of AAV5-hFIXco-Padua \\
\hline
\end{tabular}

\section{Figure legends}

Figure 1. ODs for the treatment of Haemophilia A and B (n. 41).

Figure 2. Haemophilia 'active' ODs classification according to MOA (n. 26).

Figure 3. Mechanisms of action of novel non-factor therapeutics for haemophilia. Haemostatic nonfactor agents in varying phases of development include substitution therapies (Emicizumab) for FVIII that an restore factor Xa generation and rebalancing therapies, which knock down or disrupt the natural anticoagulants (small interfering RNA to AT and PS, monoclonal antibodies to TFPI), to augment haemostasis. 


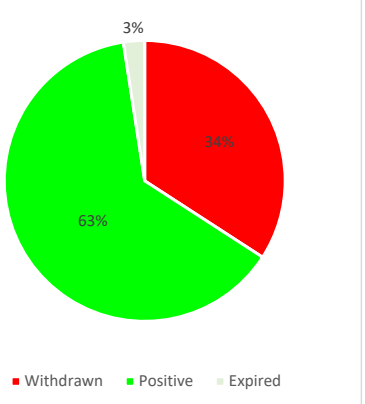




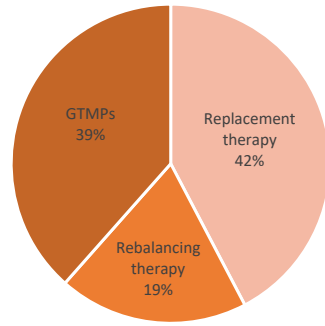




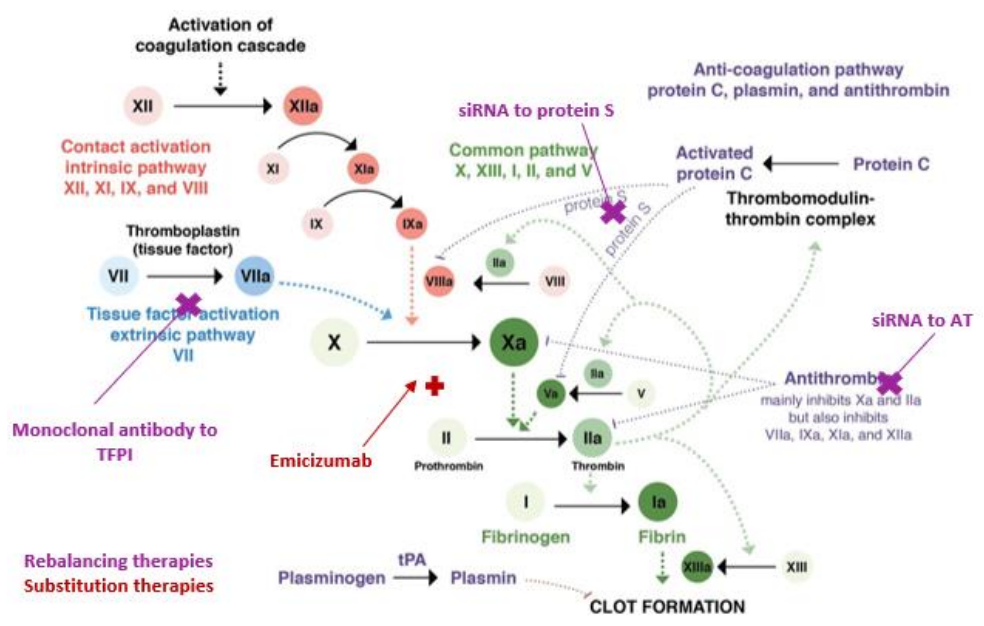

